\title{
Testing Dentists' General Sleep Knowledge With the ASKME Survey
}

\author{
Michael Simmons DMD, MSc, MPH; James Sayre, DrPH, MS \\ UCLA Fielding School of Public Health, Los Angeles, California
}

\begin{abstract}
Objective: Unhealthy sleep is an unmet public health problem with insufficient primary care providers identifying and treating sleep health problems in the general population. This research investigated the general sleep knowledge of two groups of dentists using the questions from a validated questionnaire. Results were compared to peer-reviewed published studies of physician groups over the past 15 years.

Methods: The 30 general sleep knowledge questions in the Assessment of Sleep Knowledge in Medical Education (ASKME) survey were dispersed randomly within a larger sleep-focused questionnaire that included demographics, education hours, and self-perceived expertise in sleep, given to two groups of California dentists. Group 1 responded remotely using an electronic questionnaire and group 2 were in a classroom setting receiving the same questionnaire in written form. Comparable physician groups in peer-reviewed studies were found through PubMed and Google Scholar.

Results: The overall correct score for the ASKME survey was $48.1 \%$ for dentist group $1, \mathrm{~N}=107$ and $47.4 \%$ for dentist group 2 , $\mathrm{N}=$ 63. By comparison, the correct score for the ASKME Survey was $47.9 \%$ for the physician group $\mathrm{N}=223$. Specific ASKME survey questions were answered correctly more frequently by each of the physician and dentist groups $(P<0.017)$. Approximately 100 hours of education in sleep was needed for dentist participants to self-assess as competent in sleep health knowledge.

Conclusions: Dentist and physician groups are lacking in general sleep knowledge. Dentists may have noninferior general knowledge in sleep health when compared to primary care physicians, supporting dentists' public health role as primary sleep health providers and suggesting dentists should be encouraged in this role.
\end{abstract}

Keywords: ASKME, dental education, sleep knowledge, sleep knowledge testing, sleep medicine education

Citation: Simmons M, Sayre J. Testing dentists' general sleep knowledge with the ASKME survey. J Dent Sleep Med. 2020;7(4)

\section{INTRODUCTION}

Sleep health addresses two main components: the medical issues surrounding sleep disorders and the behavioral issues associated with sleep sufficiency. Unhealthy sleep continues to be an enormous unmet public health problem in the United States, ${ }^{1}$ a hyperendemic trend that is worsening both in the United States and internationally. ${ }^{2,3}$ Although healthy sleep, along with healthy diet and exercise, contribute to longevity and quality of life, the general lack of education about healthy sleep continues to be prevalent in health care providers and this has been confirmed through testing the sleep knowledge of primary care providers (PCP's) with various questionnaires. $^{4-7}$ How well PCPs perform on questionnaires depends on educational hours both in their degree programs and subsequently. Studies of educational hours spent on sleep-related topics within US medical (physician) and dental (dentist) programs have shown approximately 3 hours of total education. ${ }^{8-12}$ With this limited training, most physician and dental providers are ill-equipped to catch and manage sleep problems at the primary care level. The number of educational hours is also consistently low on an international basis ${ }^{9,13}$ and speaks to a global need to recognize the importance of sleep and sleep health, including early identification of sleep health problems by existing health care providers.
The current state of limited predoctoral sleep health education leads to little focus by health providers on their patients sleep health, which results in poor guidance for the public, who may view snoring as normal, unrestful sleep as unavoidable, and self-imposed sleep deprivation as a badge of honor.

Recognizing poor sleep as a public health problem, a groundbreaking public health initiative introduced sleep parameters to the US population in the "Healthy People 2020 " goals. ${ }^{14}$ The sleep goals included three objectives: reducing vehicle crashes due to drowsy driving; increasing the proportion of students in grade 9 through 12 and adults who get sufficient sleep; and increasing the proportion of people with obstructive sleep apnea symptoms undergoing evaluation. These goals are supported by many studies. One large 50 -state study ${ }^{15}$ found one-third of US adults are not getting enough sleep on a regular basis, resulting on average in $13 \%$ increased all-cause mortality for those with fewer than 6 hours per night of sleep compared to those with 7 to 9 hours. ${ }^{16}$ Adolescents with short sleep resulting in sleep deprivation have associated mood disorders contributing to increased suicides, sports injuries, and motor vehicle crashes, with unintentional injuries and suicides as the two leading causes of adolescent deaths in the United States. ${ }^{17,18}$ Conservative estimates on drowsy driving for all US drivers show approximately $20 \%$ of all car crashes attributable to drowsiness, with millions of 
drivers falling asleep at the wheel weekly and at least 6,000 deaths annually in the United States attributed to drowsy driving. ${ }^{19}$

Concern has been expressed regarding the scope of sleep health care provided by dentists independent of their education, experience, and credentialing. ${ }^{20}$ However, testing of sleep knowledge has repeatedly confirmed the need for more hours of predoctoral sleep health education, for both dentist and physician groups in the United States and internationally. Unfortunately, there has been little or no increase in sleep health education in medical or dental schools at the predoctoral level over the past 20 years. ${ }^{9,12}$ Even medical textbooks show no increase in text space devoted to sleep health issues over the past decade. ${ }^{21}$ Understandably, with such limited education devoted to sleep health, other sleep experts have opined that health professional education is better judged by competencybased assessment rather than degree-based entitlements. ${ }^{22}$

Published validated testing used to evaluate sleep knowledge have included the Assessment of Sleep Knowledge in Medical Education (ASKME) survey, ${ }^{4}$ focusing on general sleep knowledge; the Obstructive Sleep Apnea Knowledge and Attitude (OSAKA) questionnaire, ${ }^{6}$ focusing on sleep apnea knowledge and attitudes; OSAKA-KIDS [7] focusing on sleep apnea knowledge and attitudes related to children; and the Dartmouth Sleep Knowledge and Attitude Survey, ${ }^{5}$ focusing on sleep disorders. In addition to these tools are academic assessments on sleep knowledge, provided as part of sleep medicine credentialing through bodies such as the American Academy of Sleep Medicine, the American Academy of Dental Sleep Medicine, and medical boards such as pulmonology, neurology, psychiatry, otolaryngology, pediatrics, anesthesiology, internal medicine and family medicine.

This study used one of the general sleep knowledge tests, the ASKME survey, because it was readily available in the literature and had been used on both physician and dentist populations in the recent past, including both students and those in primary care, as well as pharmacists and physical therapists. ${ }^{23-30}$ Although most of the published studies using the ASKME survey are not on US populations, the ASKME survey was first developed and validated in the United States. ${ }^{4}$

The ASKME survey is a validated questionnaire with internal consistency and reliability and was developed in $2001 .^{4}$ It is a 30 -item questionnaire testing 5 subset areas of sleep: basic sleep principles; circadian sleep/wake cycles; sleep architecture; common sleep disorders; and the effects of drugs and alcohol on sleep. The answer options are True, False, or Don't Know and only correct answers are included in the total score. Although the subsets of questions within the ASKME are useful, the individual questions provide more insight into specific gaps and strengths or competency in sleep knowledge. Previously, no peer-reviewed publication has assessed the specific knowledge gaps. In this study, consistent with past literature, it was determined that when at least $80 \%$ of participants answered the question correctly it indicated sufficiency or competence in that sleep topic, but when fewer than $60 \%$ of the participants answered a question correctly it indicated a gap in knowledge. An alternative method to identify gaps in sleep knowledge was used in this study and was used when at least $25 \%$ of participants responded "don't know" or chose not to respond. The identified gaps can inform educators on the topics of greatest need for future educational training in sleep medicine. Using the ASKME survey provided insight as to whether both dentists and physicians have equivalent general knowledge of sleep, as well as where specific knowledge sufficiency and gaps exist.

\section{METHODS}

General sleep knowledge was collected on two dentist groups located in California and compared for consistency in correctly answered questions and total correct score. For group 1, 110 consenting participants were recruited through advertisements in two leading California dentist publications: the California Dental Association (CDA) journal and CDA update. Monthly advertisements encouraged readers to visit a website, to take the dental sleep questionnaire containing the ASKME survey, placed in the January, February, and March 2019 editions for a total of 6 advertisements, each with the potential to reach approximately 27,000 recipient dentists. Data collection was conducted between January 1 through April 30, 2019.

For group 2 participants, CDA regional directors were contacted and their help requested to recruit study participation for their membership. To encourage participation the individual dentist participants were given a free 4-hour sleep education training following completion of their "in person" hand-written dental sleep questionnaire containing the ASKME survey. There was a total of 63 participants from group 2, which included 35 participants from Northern California and 28 from Southern California. Informed consent was obtained by all participants prior to participation in this study.

For the assessment tool, the ASKME survey questions were randomly distributed among other sleep-related questions in a sleep questionnaire containing a total of 140 questions. Of the 55 total sleep knowledge questions, 30 were ASKME survey questions. There were also 12 demographic questions, 18 attitude questions, and the remaining 55 questions were behavioral questions related to sleep assessments and therapies applied by participants in their dental practice. A few ASKME survey questions were not answered by participants and these questions were scored as incorrect when determining the percentage correct for that question. This study addressed both the total score for each of the two dental groups and individual question scores to look for gaps in knowledge. When at 
least $80 \%$ of participants answered a specific question correctly, it was indication of adequate education in this domain; where fewer than $60 \%$ of participants answered a question correctly, it indicated a significant gap in knowledge in that domain. Within the two dental groups the number of "don't know" answers were also tallied because each question where at least $25 \%$ of "don't know" or no response answers by participants was an additional way to identify gap areas of knowledge. Analyses of "don't know" responses have not been critically examined in peerreviewed literature on a regular basis and were not available in previously published reports using the ASKME survey. This research also sought to identify disparities between the physician and dentist groups in comparing areas of sufficient knowledge and knowledge gaps.

Following the collection of answers from the dentist participants, a comparative analysis was made between this survey data and previously published physician and dentist groups that used the ASKME survey. Search engines PubMed and Google Scholar were used with search criteria including all peer-reviewed published studies since 2005 using word search criteria of "ASKME, Sleep Knowledge and Sleep Knowledge Questionnaire". Several physician groups had published results without details of percentage correct by individual question and were excluded from the comparative analysis of individual questions.

Percentage of correct answers and sample sizes for each of the study questions were entered into MS Excel spreadsheets and chi-square statistical tests were performed using Stata v.15 statistical software (StataCorp LLC, College Station, Texas). The statistical significance between scores on individual questions and total correct score was evaluated using a Bonferroni adjustment. The adjusted significance value was determined to be $P<0.017$.

\section{RESULTS}

For dentist group 1, there were 110 participants who started the survey, of whom three failed to reach the halfway point and were considered to have dropped out. This left 107 participants included in the study. The age range in participants was 53.6 years (range 27-86) and average years in dental practice was 26.8 (range 0-68), where $74.2 \%$ were general dentists and $57.8 \%$ received their degree in dentistry in California dental schools.

For dentist group 2 there were 63 consenting dentists and all 63 participants completed the survey and were included in the study. The average age for dentist group 2 was 53.8 years (range 27-79) with average years in practice as a DDS as 25 (range $1-52$ ); $74.5 \%$ of participants were general dentists and $59.4 \%$ had received their degree in dentistry from California schools. Demographic data between the two dental groups were compared, indicating no significant differences between the two dental groups in terms of age, years in practice as a dentist, self-perceived level of expertise, correct responses to individual ASKME survey questions and ASKME survey total scores.

Table 1 shows the percentage distribution of participants by decade age and Table 2 the percentage distribution of participants by years in practice. Group 2 was a composite of two subgroups: one from northern California $(\mathrm{N}=35)$ and the other from southern California $(\mathrm{N}=28)$. Education hours in sleep medicine after graduating dental school were tabulated and cross referenced in Table 3 with self-perceived level of expertise as a Likert 5-point scale ranging from novice (1) to beginner (2), to competent (3) to expert (4) to thought leader (5).

For dentist group 1 (online), there was a range of responses between 96 and 110 for each of the 30 ASKME survey questions that averaged 104 answers. For dentist group 2 (in person) participants there were 63 respondents for all questions. Dentist group 1 was analyzed using the online Qualtrics survey instrument whereas dentist group 2 in-person results were taken from the paper survey instrument and entered into an Excel spreadsheet for analysis.

Using PubMed and Google Scholar search engines, four published studies met criteria for publication in the past 15 years for physician and dentist groups (Table 3 ). Of these articles only one study included practicing physicianparticipants. Three of the four studies were from cohorts that included at least $75 \%$ of predoctoral physician participants. Although the predoctoral physician groups answered all 30 questions in the ASKME survey and are reported in Table 3 as comparison groups, the dentist predoctoral training group only included 9 of the 30 ASKME survey questions; accordingly, it was deemed too limited in scope of ASKME survey questions for comparison and could not be total score-matched because they missed 21 ASKME survey questions [29]. One study of a practicing physician group of 223 subjects, conducted in the Kingdom of Saudi Arabia (KSA), met all of the inclusionary research criteria as well as listing the percentage of correct scores for all 30 individual ASKME survey questions [22].

Outcomes for both dentist groups 1 and 2 were compared to the four previously published physician groups that met the inclusionary criteria for ASKME survey total score comparison in Table 3. Additional comparison was made to the one physician group that listed individual question scoring in Table 4. Figure 1 lists percentages of correct scores for each of the 30 ASKME survey questions and significance values.

There was no significant difference in the total ASKME survey scores between the two dentist groups and the physician groups, although there was at least 10 more years of practice for both dentist groups compared to the physician group (Table 3).

Although total ASKME scores by the physician and both dentist groups were similar, individual questions were 
Table 1. Percentage of participants by decade age group.

\begin{tabular}{|l|l|l|l|l|l|l|l|l|}
\hline Group & Age range (y) & $<30$ & $30-39$ & $40-49$ & $50-59$ & $60-69$ & $70-79$ & $80-89$ \\
\hline Dental 1 & \% of participants & 1.9 & 14 & 19.6 & 23.4 & 28.0 & 11.2 & 11.9 \\
\hline Dental 2 & \% of participants & 3.2 & 12.9 & 19.4 & 33.9 & 22.6 & 8.1 & 0 \\
\hline
\end{tabular}

Table 2. Percentage of participants by years of practice of dentistry.

\begin{tabular}{|l|l|l|l|l|l|l|l|}
\hline Year of practice & $<10$ & $10-19$ & $20-29$ & $30-39$ & $40-49$ & $50-59$ & $60-69$ \\
\hline \% Group 1 & 12.4 & $1=18.1$ & 19 & 35.2 & 11.3 & 1 & 1.9 \\
\hline \% Group 2 & 13.1 & 16.4 & 31.1 & 23.0 & 9.8 & 6.6 & 0 \\
\hline
\end{tabular}

Table 3. Comparison of the two dental groups to past physician groups.

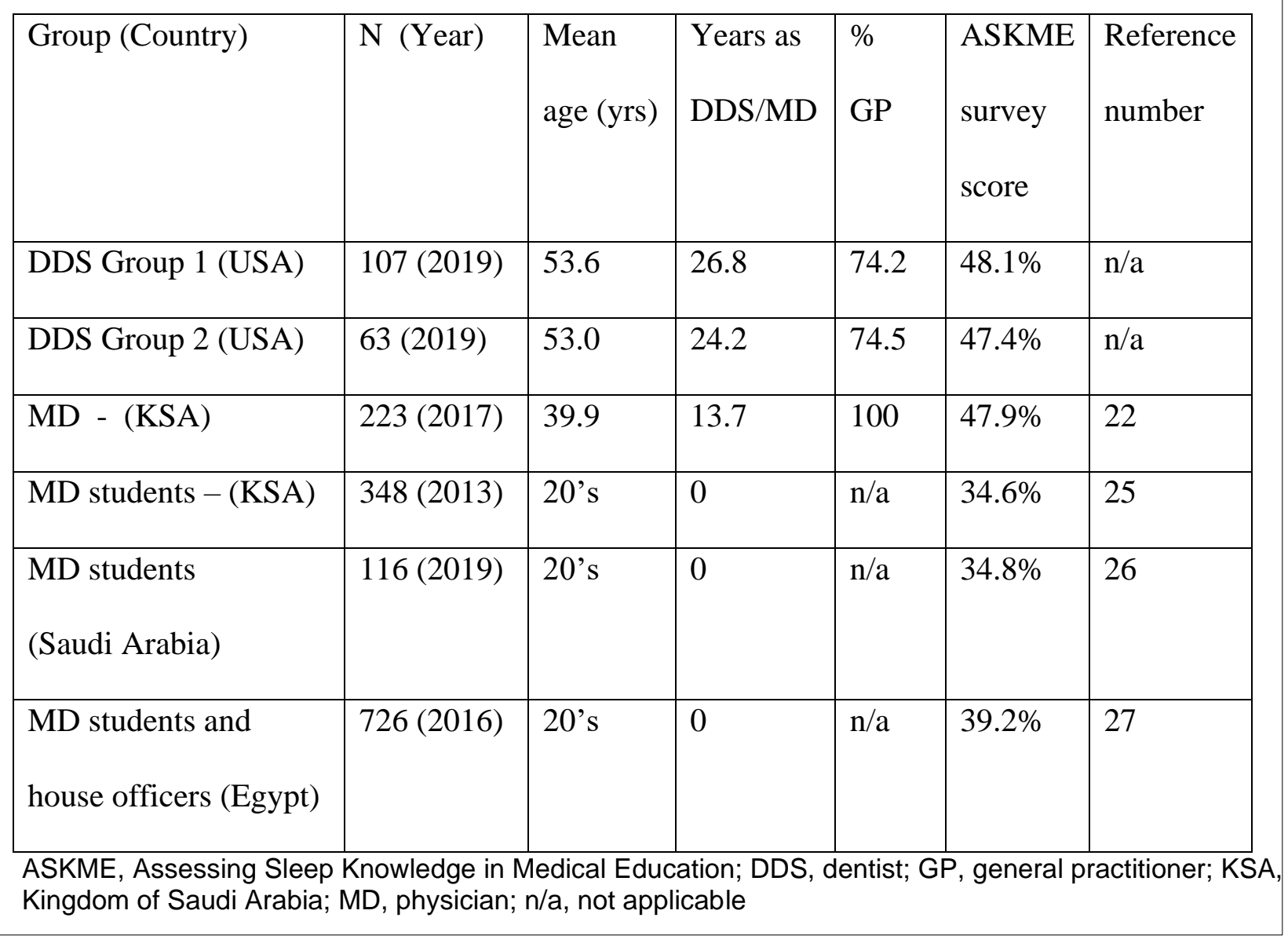

compared to one of the four comparison papers that included the percentage of correct answers by each of the 30 ASKME survey questions. This comparison was entered into an Excel spreadsheet and analyzed by a statistician for significance, using $\mathrm{P}=0.017$ values as significant. In comparing the groups, the number of respondents as well as percentage of correct answers for each question were included in the statistical comparison for significance.

A metric of at least $80 \%$ correct was used to determine group competence in a knowledge area. When comparing groups for competence in their answers to individual questions, the physician group had only one question answered correctly by at least $80 \%$ of its group participants. This question, \#5, related to the number of daily hours of sleep needed by a newborn, was also answered correctly by at least $80 \%$ in both dentist groups. Both dentist groups answered correctly four additional questions in common by at least $80 \%$ of their respective dentist groups (Table 4). This result indicated both dentist groups had consistency within their additional areas of 
Table 4. Individual ASKME survey questions answered according to group and looking for significant differences in percentage of correct scores when comparing physician to two dentist groups 1 and 2). (See Figure 1 for absolute scores)

\begin{tabular}{|c|c|c|c|c|c|c|}
\hline $\mathrm{Q} \#$ & Question pertains to & MD & \multicolumn{2}{|c|}{$P<0.017$} & \multirow{2}{*}{$\frac{\text { DDS } 2}{<\mathbf{6 0 \%}}$} & $P<0.017$ \\
\hline 1 & The need for sleep & $<60 \%$ & $<60 \%$ & & & \\
\hline 2 & Melatonin & & ***** & DDS & **** & DDS \\
\hline 3 & REM sleep activity & $<60 \%$ & $<60 \%$, DK & & & \\
\hline 4 & Weekend sleeping & $<60 \%$ & & DDS & & DDS \\
\hline 5 & Newborns sleep & $* * *$ & $* * *$ & & $* * *$ & \\
\hline 6 & Insomnia frequency & $<60 \%$ & $<60 \%$, DK & & $<60 \%$, DK & \\
\hline 7 & Preadolescent sleep onset & $<60 \%$ & $<60 \%$ & & $<60 \%$ & $\mathrm{MD}$ \\
\hline 8 & Narcolepsy onset & & $<60 \%, \quad$ DK & & $<60 \%$, DK & \\
\hline 9 & Ability to sleep & & $* * *$ & DDS & $* * *$ & DDS \\
\hline 10 & Slow wave sleep occurrence & $<60 \%$ & $<60 \%$, DK & & $<60 \%$, DK & $\mathrm{MD}$ \\
\hline 11 & Amounts of slow wave sleep & $<60 \%$ & $\langle 60 \%$, DK & & $\langle 60 \%$, DK & \\
\hline 12 & Sleepwalking & $<60 \%$ & $<60 \%$, DK & MD & $<60 \%$, DK & $\mathrm{MD}$ \\
\hline 13 & Episodes of REM & $<60 \%$ & $<60 \%$, DK & & $<60 \%$, DK & \\
\hline 14 & Periodic limb movements & $<60 \%$ & $<60 \%$, DK & & $<60 \%$ & \\
\hline 15 & Childhood hyperactivity & & $* * *$ & DDS & $* * *$ & \\
\hline 16 & Alcoholics and sleep & $<60 \%$ & $<60 \%$, DK & & $<60 \%$, DK & \\
\hline 17 & Daytime napping & $<60 \%$ & $<60 \%$ & DDS & $<60 \%$, DK & \\
\hline 18 & Weight loss and sleep apnea and snoring & & $* * *$ & & $* * *$ & \\
\hline 19 & Slow wave sleep and exercise & $<60 \%$ & $<60 \%$, DK & & $<60 \%$, DK & \\
\hline 20 & Bedwetting treatment & $<60 \%$ & $<60 \%$, DK & $\mathrm{MD}$ & $<60 \%$, DK & $\mathrm{MD}$ \\
\hline 21 & Nightmares during the sleep cycle & $<60 \%$ & $<60 \%$, DK & & $<60 \%$, DK & \\
\hline 22 & Heart rate, respiration, blood pressure, and REM & & $<60 \%, \quad \mathrm{DK}$ & & $<60 \%$, DK & MD \\
\hline 23 & Medications causing sleep difficulties & & $<60 \%, \quad \mathrm{DK}$ & MD & $<60 \%$, DK & MD \\
\hline 24 & Age and early-morning awakenings & & $<60 \%, \quad \mathrm{DK}$ & MD & $\mathrm{DK}$ & \\
\hline 25 & Alcohol and jet lag & $<60 \%$ & $* * *$ & DDS & & DDS \\
\hline 26 & Night shift workers and sleep & & $<60 \%$ & & & \\
\hline 27 & Sleepwalking and REM & $<60 \%$ & $<60 \%$, DK & & $<60 \%$ & \\
\hline 28 & Menopausal women and sleep apnea & & $<60 \% \mathrm{DK}$ & & & \\
\hline 29 & Irregular sleep and children & $<60 \%$ & $<60 \%$, DK & & $<60 \%$, DK & \\
\hline 30 & Narcolepsy and the brain & $<60 \%$ & $<60 \%, \quad$ DK & MD & $<60 \%$, DK & \\
\hline \multicolumn{7}{|c|}{$\begin{array}{l}\text { *** indicates "competency" good knowledge with }>80 \% \text { of the group having the correct answer. } \\
<60 \%=<60 \% \text { correct within the group tested (a knowledge gap - using criteria } 1) . \\
\text { DK = group answered "don't know" }>25 \% \text { (a knowledge gap - using criteria } 2) . \\
\text { MD = Significantly higher score in this group }(P<0.017) \text { level by MD when comparing MD and DDS groups. } \\
\text { DDS = Significantly higher score in this group }(P<0.017) \text { level by DDS when comparing MD and DDS groups. } \\
\text { Blank cell indicates the group scored above } 60 \% \text { but less than } 80 \% \text { correct and less than } 25 \% \text { were DK. } \\
\text { Bold print question topics indicate gap areas of knowledge across all three groups. } \\
\text { REM, rapid eye movement. }\end{array}$} \\
\hline
\end{tabular}

knowledge competence. The four additional questions in common for both dentist groups were on topics related to melatonin, ability to sleep, childhood hyperactivity, and weight loss as it relates to snoring and sleep apnea.

Knowledge gaps were identified by two criteria. The first criterion is that fewer than $60 \%$ of the group scored correctly on an individual question. The second criterion is whether "don't know" answers were chosen by at least $25 \%$ of responding participants. These "don't know" data points were only available for the dental groups. Dentist group 1 had at least 25\% responding "don't know" to 19 questions and dentist group 2 to 18 questions. There was considerable overlap between both dentist groups regarding the "don't know" responses by at least $25 \%$ of participants; both dentist groups gave this response to the same 17 questions (Table 4).

In total, approximately two-thirds of the ASKME survey questions were not answered correctly by more than $60 \%$ of participants for each group (19/30 for physician and dentist group 2 and 23/30 for dentist group 1) Questions 22 and, 23 were answered correctly by more than $60 \%$ of the physician group but were knowledge gaps by both dentist groups. The topics covered in these questions were, related to medication effects on sleep and rapid eye movementrelated heart rate/respiration/blood pressure. These topic areas could be specifically targeted for future dentist 
Table 5. Self-perceived expertise in sleep health cross referenced by dentist postdoctoral hours of education in sleep.

\begin{tabular}{|c|c|c|c|c|c|c|c|c|c|c|c|c|}
\hline \multirow{2}{*}{\begin{tabular}{|l}
$\begin{array}{l}\text { Hours of } \\
\text { education }\end{array}$ \\
Group
\end{tabular}} & \multicolumn{2}{|c|}{ Total N (\%) } & \multicolumn{2}{|l|}{ Novice } & \multicolumn{2}{|c|}{ Beginner } & \multicolumn{2}{|c|}{ Competent } & \multicolumn{2}{|c|}{ Expert } & \multicolumn{2}{|c|}{ Thought leader } \\
\hline & 1 & 2 & 1 & 2 & 1 & 2 & 1 & 2 & 1 & 2 & 1 & 2 \\
\hline $\begin{array}{l}\text { Total } \\
\text { count }\end{array}$ & 95(100) & $59(100)$ & 33(35) & $22(37)$ & 33(35) & $30(51)$ & $27(28)$ & $6(10)$ & 1(1) & 0 & 1(1) & $1(2)$ \\
\hline $\mathbf{0}$ & $13(14)$ & 11(19) & 12(13) & $6(10)$ & 1(1) & $5(8)$ & & & & & & \\
\hline $4-9$ & $14(15)$ & $11(19)$ & $8(8)$ & $4(7)$ & $6(6)$ & $7(12)$ & & & & & & \\
\hline $10-24$ & $24(25)$ & $13(22)$ & $5(5)$ & $2(3)$ & 17(18) & $11(19)$ & $2(2)$ & & & & & \\
\hline $25-64$ & $20(21)$ & $5(8)$ & $3(3)$ & & 9(9) & $3(5)$ & $8(8)$ & $2(3)$ & & & & \\
\hline $65-99$ & $8(8)$ & $2(3)$ & 1(1) & & $2(2)$ & & $5(5)$ & $2(3)$ & & & & \\
\hline$>1000$ & $2(2)$ & 0 & & & & & $1(1)$ & & & & 1(1) & \\
\hline
\end{tabular}

$\mathrm{N}$ is the total number of dentists who responded to this question.

Figure 1. Significant differences between MD (physician) and two DDS (dentist) groups in answering individual ASKME questions. Absolute scores listed as percent correct for each question.

\begin{tabular}{|c|c|c|c|c|c|c|}
\hline 1 & 40.4 & 34.6 & 0.3108 & 44.4 & 0.5691 & 0.2041 \\
\hline 2 & 64.1 & 86.8 & $<.0001$ & 82.5 & 0.0056 & 0.4457 \\
\hline 3 & 55.2 & 53.3 & 0.7456 & 65.1 & 0.1606 & 0.1326 \\
\hline 4 & 51.6 & 69.2 & 0.0025 & 77.8 & 0.0002 & 0.2258 \\
\hline 5 & 84.3 & 85 & 0.8693 & 87.3 & 0.5564 & 0.6777 \\
\hline 6 & 31.4 & 30.8 & 0.9123 & 33.3 & 0.775 & 0.7352 \\
\hline 7 & 58.7 & 46.7 & 0.0407 & 38.1 & 0.0038 & 0.2747 \\
\hline 8 & 34.5 & 29.9 & 0.4055 & 30.2 & 0.5233 & 0.9781 \\
\hline 9 & 63.2 & 84.1 & 0.0001 & 85.7 & 0.0007 & 0.7796 \\
\hline 10 & 31.4 & 19.6 & 0.0247 & 11.1 & 0.0013 & 0.1488 \\
\hline 11 & 22.9 & 28 & 0.3137 & 11.1 & 0.0398 & 0.0099 \\
\hline 12 & 24.7 & 21.5 & 0.522 & 9.5 & 0.0093 & 0.0445 \\
\hline 13 & 34.1 & 41.1 & 0.216 & 38.1 & 0.5566 & 0.6998 \\
\hline 14 & 38.1 & 32.7 & 0.3389 & 33.3 & 0.486 & 0.9359 \\
\hline 15 & 76.2 & 91.6 & 0.0008 & 81 & 0.4222 & 0.0423 \\
\hline 16 & 25.1 & 15 & 0.0376 & 14.3 & 0.0709 & 0.901 \\
\hline 17 & 34.1 & 59.8 & $<.0001$ & 33.3 & 0.9057 & 0.0008 \\
\hline 18 & 77.6 & 87.9 & 0.0258 & 87.3 & 0.0908 & 0.9085 \\
\hline 19 & 44.4 & 43.9 & 0.9318 & 58.7 & 0.0448 & 0.0479 \\
\hline 20 & 44.4 & 14 & $<.0001$ & 12.7 & $<.0001$ & 0.8108 \\
\hline 21 & 34.1 & 26.2 & 0.1483 & 39.7 & 0.4116 & 0.0665 \\
\hline 22 & 67.7 & 54.2 & 0.0172 & 50.5 & 0.0121 & 0.6407 \\
\hline 23 & 61.4 & 41.1 & 0.0005 & 41.3 & 0.0044 & 0.9796 \\
\hline 24 & 68.2 & 53.3 & 0.0085 & 65.1 & 0.6427 & 0.1326 \\
\hline 25 & 38.6 & 83.2 & $<.0001$ & 61.9 & 0.001 & 0.0019 \\
\hline 26 & 72.2 & 59.8 & 0.0236 & 68.3 & 0.5455 & 0.2677 \\
\hline 27 & 26.5 & 25.2 & 0.8012 & 22.2 & 0.4896 & 0.6587 \\
\hline 28 & 60.5 & 57.9 & 0.6522 & 71.4 & 0.1138 & 0.0784 \\
\hline 29 & 52.9 & 56.1 & 0.5851 & 54 & 0.8772 & 0.7902 \\
\hline 30 & 19.7 & 9.3 & 0.0167 & 12.7 & 0.2031 & 0.486 \\
\hline
\end{tabular}

Blue cells indicate MD correct score was significantly better than DDS score $P<0.017$

Green cells indicate DDS correct score was significantly better than MD score $P<0.017$

Yellow cells indicate significance between both DDS groups $\mathrm{P}<0.017$

MD comparison scores from published reference \#23 
education. Questions 4 and 25 were answered correctly by fewer than $60 \%$ of the physicians but neither of the dentist groups. The topics of these questions addressed weekend sleeping and effects of alcohol/jet lag on sleep. For the 30 questions there were a total of 90 opportunities to identify knowledge gaps and areas of knowledge competence in the three comparison groups. Results indicate that 69\% (62/90) of the group answers reflected knowledge gaps and 13\% $(12 / 90)$ of the group answers reflected knowledge competency.

In the two dental groups, hours of education in postdoctoral education were related to the self-evaluation of expertise (Table 5). Results indicated that fewer than one-fourth (36 of 154 or $23 \%$ ) of the dentist participants who answered the self-assessment of expertise question felt competent or more expert with sleep health, and this appears related to the number of hours of education. Approximately $2 \%$ of dentists (3/154) reported themselves as expert or better at sleep medicine and $1.3 \%(2 / 154)$ reported they had received more than 1,000 hours of postdoctoral education in sleep topics. Self-assessment of competency in sleep medicine was achieved with $100 \%$ $(17 / 17)$ of dentist responders having a minimum of 100 hours of sleep education, $70 \%(7 / 10)$ of dentists with 65 to 99 hours of sleep education, and $40 \%(10 / 25)$ of dentists with 25 to 64 hours of sleep education. This would seem reasonable given that only 3 hours of education is typically received in dental or medical doctorate degree programs.

\section{DISCUSSION}

Approximately one-third of the ASKME survey questions were answered correctly by more than $60 \%$ of participants in both the physician and dentist groups, confirming large knowledge gaps by these groups in addressing population sleep health. Similarly, between one- half and two-thirds of dentist participants had a gap in knowledge indicated by $25 \%$ or more respondents answering "don't know" responses to specific questions. This study confirms that the two dentist groups lack general sleep knowledge consistent with past reports that previously evaluated physician groups using the same ASKME survey questions. ${ }^{22,25-27}$ The main comparison in this study was the two California dentist groups of 170 total participants with a physician group of 223 participants from KSA. ${ }^{22}$ Although this KSA cohort may appear not to not reflect US physician general sleep knowledge, the KSA physician population was identified as having 2.6 hours (156 minutes) of education in sleep medicine, which is similar to the international average of 146 minutes for physicians, albeit lower than the 187 minutes reported for US physician. ${ }^{9}$

An interesting finding was the relationship in the dentist groups between hours of education in sleep and selfperceived level of expertise. One hundred percent of dentist participants self-assessed themselves as competent in sleep medicine with a minimum of 100 hours of education (range 100-249 hours) versus $70 \%$ of responders self-assessing competency with a minimum of 65 hours of education in sleep (range 65-99 hours). This would support that dentists should receive at least 100 hours of education in sleep medicine to feel competent enough to act in the role of primary care providers of sleep health.

Although this study showed no significant total score difference in general sleep knowledge between physician and dentist groups, there were significant differences in areas of strengths and gaps in general sleep knowledge between physician and dentist groups. The dentist groups had more questions answered competently (defined as a minimum of $80 \%$ correct) showing strengths (competency) in specific areas of sleep knowledge such as: topics related to melatonin, newborn sleep, ability to sleep, childhood hyperactivity, and weight loss as it relates to snoring and sleep apnea. Physicians showed knowledge strengths only in the topic of newborn sleep, although they did not have the same dentist-specific gaps in knowledge related to medication effects on sleep and rapid eye movementrelated heart rate, respiration and blood pressure.

Given the similar overall general sleep knowledge total scores using the validated ASKME survey, there is supportive rationale that dentists can act as primary care sleep health providers for population sleep health in addition to physician groups. This role for dentists may be dependent on hours of education in sleep as it relates to dentist self-assessment of competence.

There are several weaknesses in this research. Testing the sleep knowledge of only a few dentist and physician groups with a single testing questionnaire instrument may not reflect accurately on dentist and physician groups' general sleep knowledge. Californian dentist groups may not be reflective of those from other US states or dentist groups in other countries; similarly, KSA physicians may not reflect the knowledge of physicians from other countries. The results are somewhat mitigated by the consistency of the two separate dentist groups that scored very similarly using different formats of test taking. Another potential weakness of this study is bias among dentist groups in that perhaps only dentists trained in sleep would agree to participate in this study. Results showed the self-perceived level of expertise in sleep health by dentist participants included about one-third (36\%) endorsing they were novices, a little more than one-third $(41 \%)$ as beginners, and only about one-fourth $(23 \%)$ self-endorsing as competent or more expert. This implies the population of participants reflected a good spread of general expertise in sleep health among dentists and speaks against the dentist populations as skewed toward dentists with more expertise in sleep medicine. Finally, the individual questions of the ASKME survey were mixed into a larger sleep survey without ordering consistency with the original ASKME survey. It is not known whether changing the order sequence of the questions or including them in a 
larger survey questionnaire would affect the total ASKME survey score.

\section{CONCLUSION}

This study confirms a long-term and ongoing lack of knowledge in general sleep health by both physician and dentist groups. There was no significant difference in total general sleep knowledge scores between both physician and dentist groups using questions from the validated ASKME survey. Results support the need to encourage both physician and dentist providers of primary care to seek ongoing postdoctoral education in sleep health in order to attain self-efficacy in sleep health. Results of this research also speak to specific sleep topic areas to address knowledge gaps. At least 100 hours of postdoctoral sleep education may be a worthy goal in order to achieve a selfassessment by dentists for competence in sleep health.

The recruitment and encouragement of more primary care health professionals, both dentists and physicians, to act in the capacity of primary sleep health providers in the diagnosis and treatment of noncomplex sleep health problems can help mitigate the enormous gaps in diagnosis and treatment of population-level sleep disorders and sleep sufficiency.

\section{ABBREVIATIONS}

ASKME, Assessment of Sleep Knowledge in Medical

Education

CDA, California Dental Association

KSA, Kingdom of Saudi Arabia

PCP, primary care physician

\section{REFERENCES}

1. Institute of Medicine (US) Committee on Sleep Medicine and Research. Sleep Disorders and Sleep Deprivation: An Unmet Public Health Problem. Colten HR, Altevogt BM, eds. Washington, DC: National Academies 2006. http://www.ncbi.nlm.nih.gov/books/NBK19960/. Accessed July 14, 2018.

2. Peppard PE, Young T, Barnet JH, Palta M, Hagen EW, Hla KM. Increased prevalence of sleep-disordered breathing in adults. Am J Epidemiol. 2013;177(9):1006-1014. doi:10.1093/aje/kws342

3. Ford ES, Cunningham TJ, Giles WH, Croft JB. Trends in insomnia and excessive daytime sleepiness among U.S. adults from 2002 to 2012. Sleep Med. 2015;16(3):372-378. doi:10.1016/j.sleep.2014.12.008

4. Zozula R, Bodow M, Yatcilla D, Cody R, Rosen RC. Development of a brief, self-administered instrument for assessing sleep knowledge in medical education: "the ASKME Survey." Sleep. 2001;24(2):227233.

5. Sateia MJ, Reed VA, Christian Jernstedt G. The Dartmouth Sleep Knowledge and Attitude Survey: development and validation. Sleep Med. 2005;6(1):47-54. doi:10.1016/j.sleep.2004.07.013

6. Schotland HM, Jeffe DB. Development of the obstructive sleep apnea knowledge and attitudes (OSAKA) questionnaire. Sleep Med. 2003;4(5):443-450.
Uong EC, Jeffe DB, Gozal D, et al. Development of a measure of knowledge and attitudes about obstructive sleep apnea in children (OSAKA-KIDS). Arch Pediatr Adolesc Med. 2005;159(2):181-186. doi:10.1001/archpedi.159.2.181

8. Rosen R, Mahowald M, Chesson A, et al. The Taskforce 2000 survey on medical education in sleep and sleep disorders. Sleep. 1998;21(3):235-238

9. Mindell JA, Bartle A, Wahab NA, et al. Sleep education in medical school curriculum: a glimpse across countries. Sleep Med. 2011;12(9):928-931. doi:10.1016/j.sleep.2011.07.001

10. Simmons MS, Pullinger A. Education in sleep disorders in US dental schools DDS programs. Sleep Breath. 2012;16(2):383-392. doi:10.1007/s11325-011-0507-z

11. Mindell JA, Bartle A, Ahn Y, et al. Sleep education in pediatric residency programs: a cross-cultural look. BMC Res Notes. 2013;6:130. doi:10.1186/1756-0500-6-130

12. Ivanhoe JR, Frazier KB, Parr GR, Haywood VB. The teaching and treatment of upper airway sleep disorders in North American dental schools. J Prosthet Dent. 2003;89(3):292-296. doi:10.1067/mpr.2003.52

13. Balasubramaniam R, Pullinger, A, Simmons, M. Sleep medicine education at dental schools in Australia and New Zealand. $J$ Dent Sleep Med. 2014;2(1):9-16.

14. Sleep Health $\mid$ Healthy People 2020 https://www.healthypeople.gov/2020/topics-objectives/topic/sleephealth. Accessed September 18, 2020.

15. Liu Y, Wheaton AG, Chapman DP, Cunningham TJ, Lu H, Croft JB Prevalence of healthy sleep duration among adults--United States, 2014. MMWR Morb Mortal Wkly Rep. 2016;65(6):137-141. doi:10.15585/mmwr.mm6506al

Hafner M, Stepanek M, Taylor J, Troxel WM, van Stolk C. Why sleep matters - the economic costs of insufficient sleep. Rand Health $Q$. 2017;6(4).

17. Shekari Soleimanloo S, White MJ, Garcia-Hansen V, Smith SS. The effects of sleep loss on young drivers' performance: A systematic review. PLoS One. 2017;12(8): 0184002.

18. Heron M. Deaths: Leading Causes for 2017. National Vital Statistics Reports, Vol. 68, No. 6, June 24, 2019.

19. Higgins JS, Michael J, Austin R, et al. Asleep at the wheel-the road to addressing drowsy driving. Sleep. 2017;40(2) doi:10.1093/sleep/zsx001

20. Quan SF, Schmidt-Nowara W. The role of dentists in the diagnosis and treatment of obstructive sleep apnea: consensus and controversy. J Clin Sleep Med. 2017;13(10):1117-1119. Published 2017 Oct 15. doi: $10.5664 /$ jcsm. 6748

21. Teodorescu MC, Avidan AY, Teodorescu M, et al. Sleep medicine content of major medical textbooks continues to be underrepresented. Sleep Med 2007; 8:271-276.

22. Strohl KP. Sleep medicine training across the spectrum. Chest. 2011; 139(5):1221-1231

23. Saleem AH, Al Rashed FA, Alkharboush GA, et al. Primary care physicians' knowledge of sleep medicine and barriers to transfer of patients with sleep disorders. A cross-sectional study. Saudi Med J. 2017;38(5):553-559.

24. Tze-Min Ang K, Saini B, Wong K. Sleep health awareness in pharmacy undergraduates and practicing community pharmacists. $J$ Clin Pharm Ther. 2008;33(6):641-652.

25. Talaat W, AlRozzi B, Kawas SA. Sleep medicine education and knowledge among undergraduate dental students in Middle East universities. Cranio. 2016;34(3):163-168.

26. Almohaya A, Qrmli A, Almagal N, et al. Sleep medicine education and knowledge among medical students in selected Saudi medica schools. BMC Med Educ. 2013;13:133. 
Knowledge and attitude regarding sleep medicine among medical students at Qassim University, Saudi Arabia. Open Access Maced J Med Sci. 2019;7(17):2895-2901.

28. Zaki NWF, Marzouk R, Osman I, Alamah HY, et al. Sleep medicine knowledge among medical students in seven Egyptian medical faculties. J Sleep Disord Ther. 2016;5:2.

29.

Ume-Aiman, Nabi N, Sadiq S. Knowledge and attitude of physical therapists towards sleep quality assessment. $J$ Adv Medicine Medical Res. 2019;28(6):1-9. https://doi.org/10.9734/JAMMR/2018/45374.

30. Talaat W, AlRozzi B, Kawas SA. Sleep medicine education and knowledge among undergraduate dental students in Middle East universities. Cranio. 2016;34(3):163-168. doi:10.1179/2151090315Y.0000000019

\section{SUBMISSION \& CORRESPONDENCE INFORMATION}

Submitted for publication June 18, 2020

Accepted for publication August 26, 2020

Address correspondence to: Michael Simmons, DMD, MSc, MPH, Director, Encino Center for Sleep and TMJ

Disorders: 16500 Ventura Blvd. Ste 370, Encino, CA 91436; Email: msimmons@g.ucla.edu

\section{DISCLOSURE STATEMENT}

The authors have no conflicts of interest to disclose. 